\title{
A Case of Poorly Differentiated Hepatocellular Carcinoma, Lymphocyte-rich.
}

\author{
Ginji Omori ${ }^{1}$, Takahiro Osuga ${ }^{1}$, Koji Miyanishi ${ }^{1}$, Kota Hamaguchi ${ }^{1}$, Shingo Tanaka ${ }^{1}$, \\ Hiroyuki Ohnuma $^{1}$, Kazuyuki Murase ${ }^{1}$, Kohichi Takada ${ }^{1}$, Minoru Nagayama ${ }^{1}$, Yasutoshi \\ Kimura $^{1}$, Ichiro Takemasa ${ }^{1}$, Yasuhiro Kikuchi ${ }^{1}$, Toshihiko Torigoe ${ }^{1}$, and Junji Kato ${ }^{1}$ \\ ${ }^{1}$ Sapporo Medical University
}

June 4, 2021

\begin{abstract}
A 70-year-old woman was diagnosed poorly differentiated hepatocellular carcinoma (HCC), lymphocyte rich. In this case, programmed cell death 1 expression was observed. Immune checkpoint inhibitor treatment may be effective in such cases, although there have been no reports of their use for poorly differentiated HCC, lymphocyte-rich.
\end{abstract}

\section{CASE REPORT}

A case of poorly differentiated hepatocellular carcinoma, lymphocyte-rich.

Ginji Omori $^{1}$, Takahiro Osuga ${ }^{1}$, Koji Miyanishi $^{1 *}$, Kota Hamaguchi $^{1}$, Shingo Tanaka ${ }^{1,2}$, Hiroyuki Ohnuma ${ }^{1}$, Kazuyuki Murase ${ }^{1}$, Kohichi Takada ${ }^{1}$, Minoru Nagayama ${ }^{3}$, Yasutoshi Kimura $^{3}$, Ichiro Takemasa ${ }^{3}$, Yasuhiro Kikuchi $^{4}$, Toshihiko Torigoe ${ }^{4}$, Junji Kato ${ }^{1}$

${ }^{1}$ Department of Medical Oncology, Sapporo Medical University School of Medicine, Hokkaido, Japan

${ }^{2}$ Department of Infection Control and Laboratory Medicine, Sapporo Medical University School of Medicine, Hokkaido, Japan

${ }^{3}$ Department of Surgery, Surgical Oncology and Science, Sapporo Medical University School of Medicine, Hokkaido, Japan

${ }^{4}$ Department of Pathology, Sapporo Medical University School of Medicine, Hokkaido, Japan

${ }^{*}$ To whom correspondence should be addressed.

E-mail: miyako@sapmed.ac.jp

\section{Contact Information}

Koji Miyanishi, M.D., Ph.D., Department of Medical Oncology, Sapporo Medical University School of Medicine, South-1, West-16, Chuo-ku, Sapporo, Hokkaido 060-8543, Japan.

TEL: (+81)-11-611-2111(ext.32540); FAX:(+81)-11-612-7987

e-mail: miyako@sapmed.ac.jp

\section{Abstract}

A 70-year-old woman was diagnosed poorly differentiated hepatocellular carcinoma (HCC), lymphocyte rich. In this case, programmed cell death 1 expression was observed. Immune checkpoint inhibitor treatment may 
be effective in such cases, although there have been no reports of their use for poorly differentiated HCC, lymphocyte-rich.

Key Clinical Message: Pathological study in a case of poorly differentiated hepatocellular carcinoma, lymphocyte-rich suggested that immune checkpoint inhibitor may be effective. The histological type is an important factor in determining treatment choices.

Key words: hepatocellular carcinoma, hepatocellular carcinoma, lymphocyte rich, lymphoepithelial hepatocellular carcinoma, Immune checkpoint inhibitors, programmed cell death ligand 1

\section{INTRODUCTION}

In the past, poorly/undifferentiated hepatocellular carcinoma (HCC) with high lymphocytic infiltration was classified as lymphoepithelioma-like-hepatocellular carcinoma (LEL-HCC) ${ }^{1}$. In the 2019 revision of the WHO classification, it was incorporated into the lymphocyte-rich subtype, regardless of differentiation ${ }^{2}$. We experienced a case of poorly differentiated hepatocellular carcinoma (HCC), lymphocyte-rich, which corresponds to LEL-HCC in the previous classification, and performed a pathological investigation.

\section{Case Report}

In 2019, a 70-year-old woman visited her local doctor with a complaint of weight loss of $15 \mathrm{~kg}$ in a month. Abdominal ultrasound revealed a liver tumor, and she was referred to our hospital. There was no history of alcohol intake. Blood examination (Table 1) showed aspartate aminotransferase elevation (AST; 66 $\mathrm{U} / \mathrm{L}$ ), alanine aminotransferase elevation (ALT; $68 \mathrm{U} / \mathrm{L}$ ), positive HBs antigen, HBV-DNA elevation (5.6 $\operatorname{LogIU} / \mathrm{mL}$ ), and negative hepatitis C virus antibody. Alfa-fetoprotein (AFP) was markedly elevated (3946 $\mathrm{ng} / \mathrm{mL}$ ). Abdominal ultrasound revealed a $22 \times 20 \mathrm{~mm}$ mass with an internal mosaic pattern in segment 5 of the liver (Figure 1). Angiographic computed tomography (AGCT) scan revealed low absorption in CT during arterioportography (CTAP) and uniformly high absorption in both early and delayed CT during hepatic arteriography (CTHA) (Figure 2). EOB-MRI revealed a high signal in early phase, low signal in hepatocellular phase, high signal in diffusion-weighted image, and low signal in ADC (Figure 3). The lesion was solitary and there were no findings suggestive of vascular invasion. Laparoscopic partial hepatectomy was performed subsequent to the diagnosis of classical hepatocellular carcinoma (HCC) UICC Stage Ib. Microscopically, there were many infiltrating lymphocytes in the background, and tumor cells with round to oval nuclei with prominent irregularities and abundant eosinophilic sporophytes had grown in a plump, sheet-like pattern. Some tubular structures corresponding to the moderately differentiated type remained at the tumor margins; however, the majority of the cells were the poorly differentiated type (Figure 4). Immunostaining of the tumor cells was positive for glypian3 and negative for EBER. Programmed cell death ligand 1 (PD-L1) was positive in about $40 \%$ of tumor cells, and inflammatory cells around the tumor were also partially positive, showing scattered positive cells in the form of small clusters. The infiltrating lymphocytes showed a mixture of CD3- and CD8- positive cytotoxic T cells and CD4- and FOXP3-positive regulatory T cells (Figure 5-8). Twenty months have passed since hepatectomy and no recurrence has been observed to date.

\section{Discussion}

Among undifferentiated cancers with abundant lymphocyte infiltration, those derived from the head and neck region are called lymphoepithelioma ${ }^{3}$. Undifferentiated cancers with abundant lymphocyte infiltration in organs other than the head and neck are called lymphoepithelioma-like carcinomas (LELC), and in the WHO classification 4th edition, LELC in the liver were classified as lymphoepithelioma-like hepatocellular carcinomas (LEL-HCC) ${ }^{1}$. However, in the 2019 revision of the WHO classification, HCC with abundant lymphocyte infiltration was included in the classification of "hepatocellular carcinoma, lymphocyte-rich" regardless of the degree of differentiation ${ }^{2}$. The frequency of lymphocyte-rich HCC among all HCC cases is less than $1 \%$, and it is reported that its prognosis is better than that of classical $\mathrm{HCC}^{2,4}$. Most cases of LELC in other organs are associated with Epstein-Barr virus (EBV) ${ }^{5}$ Infection, but most cases of HCC, lymphocyte-rich are negative for $\mathrm{EBV}^{6}$. We searched PubMed for articles from 1995 to 2019 using the key- 
words "lymphoepithelioma-like hepatocellular carcinoma" or "hepatocellular carcinoma lymphocyte rich". As a result, there were 58 cases corresponding to poorly differentiated HCC, lymphocyte-rich (Table $2^{7-24}$ ). We examined a total of 59 cases including the current case. The median age was 60 years; 27 cases were hepatitis B virus (HBV) positive and 18 were hepatitis $\mathrm{C}$ virus (HCV) positive. EBV was negative in all but 1 case. Although there are few reports on the proportion of infiltrating lymphocyte types, there are many cases in which both CD4-positive cells, including regulatory T cells, and CD8-positive cells, considered to be cytotoxic T lymphocytes (CTLs), were present to the same extent. In this case as well, CD4 and CD8 were equally positive. FoxP3 was positive in some of the CD4-positive cells, indicating the presence of regulatory T cells.

In the IMbrave150 trial, the combination of the immune checkpoint inhibitor (ICI) atezolizumab and the angiogenesis inhibitor bevacizumab for unresectable HCC significantly prolonged overall survival compared with sorafenib. As a result, this combination therapy has come to be used clinically for unresectable HCC. The response rate for this treatment in unresectable HCC is $27.3 \%{ }^{25}$, which is a good result, but it is still necessary to identify biomarkers for predicting the effect of ICIs in terms of pharmacoeconomics and personalized medicine. In addition, Chan et al. reported that there are many mutations in the Wnt pathway, including CTNNB1, in normal HCC, as well many mutations in CCND1 in LEL-HCC. These findings suggest that LEL-HCC may have different tumor properties compared with normal $\mathrm{HCC}^{26}$. Therefore, we decided to histopathologically assess the ICI treatment strategy for poorly differentiated HCC, lymphocyte-rich. So far, there have been no reports of the use of ICIs in poorly differentiated HCC, lymphocyte-rich, but there have been multiple reports of cases in which ICIs were effective for LELC in other organs ${ }^{27,28}$. In a study of 217 cases of HCC by Calderaro et al., the PD-L1 positive rate ( $>1 \%$ of tumor cell-positive cases were defined as PD-L1 positive) of LEL-HCC tumor cells was 3 of 13 cases, which was not different from the positive rate of $17 \%$ for all HCC. However, the PD-L1 positive rate of surrounding inflammatory cells was significantly high (PD-L1 expression by inflammatory cells was classified as high if the number of PD-L1 positive clusters around the tumor was equal or superior to 6 [median of the full series]) in 12 of 13 cases $(92 \%)^{29}$. In this case as well, multiple PD-L1-positive immune cell clumps were found around the tumor (Fig. 8). It is known that ICIs induces an immune response by inhibiting PD-L1 on tumor-related macrophages ${ }^{30}$, and ICIs may be effective in poorly differentiated HCC, lymphocyte-rich. In the study by Calderaro et al., the analysis of the PD-L1 positive rate of LEL-HCC and tumor cells was limited to only 13 cases. In the present case, $40 \%$ of the tumor cells were also PD-L1 positive. There are other reports ${ }^{7}$ that have shown PD-L1 positivity in tumor cells and infiltrating lymphocytes of poorly differentiated HCC, lymphocyte-rich, which is consistent with our case. To better understand the tendency for PD-L1 expression in tumor cells in poorly differentiated HCC, lymphocyte-rich cases, it is necessary to accumulate more cases. Considering the aforementioned details, it is likely that poorly differentiated HCC lymphocyte-rich, which was once designated as LEL-HCC, can be expected to have a good therapeutic response to ICIs, and these findings may contribute to the stratification of $\mathrm{HCC}$ treatment in the future.

\section{Conclusion}

We experienced a rare case of poorly differentiated HCC, lymphocyte-rich, which may be present in HCCs showing typical imaging findings. For treatment of advanced cases, ICIs may be effective, and it was considered necessary to confirm pathological findings when selecting treatment.

\section{ACKNOWLEDGEMENTS}

All authors would like to thank the patient and his family for allowing this case study.

\section{CONFLICT OF INTEREST}

The authors have no conflict of interests.

\section{AUTHOR CONTRIBUTIONS}

GO, TO and KM: collected and analyzed data and wrote and edited the manuscript. KH, ST, HO, KT, KM and JK: involved in the patient's care. MN, YK and IT: were abdominal surgeons providing hepatectomy. 
YK and TT: were pathologists providing diagnosis.

\section{Reference}

1. Bosman FT, Carneiro F, Hruban RH, Theise ND. WHO Classification of Tumours of the Digestive System, 4th ed. 2010.

2. Classification of Tumours Editorial Board. WHO Classification of Tumours of the Digestive System, 5th ed. 2019.

3. Applebaum EL, Mantravadi P, Haas R. Lymphoepithelioma of the nasopharynx. Laryngoscope 1982; 92: 510-514.

4. Elliot DRF, Gill RM. Undifferentiated variant. In: Gastrointestinal Pathology, Edited by Gonzalez RS, Volume 13-3, 2019.

5. Iezzoni JC, Gaffey MJ, Weiss LM. The Role of Epstein-Barr Virus in Lymphoepithelioma-like Carcinomas. Am J Clin Pathol 1995; 103: 308-315.

6. Labgaa I, Stueck A, Ward SC. Lymphoepithelioma-Like Carcinoma in Liver. Am J Pathol 2017; 187: 1438-1444.

7. Putra J, Anderson TA, Roayaie S, Maeda M, Thung SN. Metastatic lymphoepithelioma-like hepatocellular carcinoma: A potential diagnostic pitfall and demonstration of Pd-l1 expression. Annals of Hepatology 2017; 16: $157-159$.

8. Shirabe K, Matsumata T, Maeda T, Sadanaga N, Kuwano H, Sugimachi K. A long-term surviving patient with hepatocellular carcinoma including lymphocytes infiltration - A clinicopathological study. Hepatogastroenterology 1995; 42: 996-1001.

9. Wada Y, Nakashima O, Kutami R, Yamamoto O, Kojiro M. Clinicopathological study on hepatocellular carcinoma with lymphocytic infiltration. Hepatology 1998; 27: 407-414.

10. Emile JF, Adam R, Sebagh M, et al. Hepatocellular carcinoma with lymphoid stroma: A tumour with good prognosis after liver transplantation. Histopathology 2000; 37: 523-529.

11. Si MW, Thorson JA, Lauwers GY, DalCin P, Furman J. Hepatocellular lymphoepithelioma-like carcinoma associated with Epstein Barr virus: A Hitherto unrecognized entity. Diagn Mol Pathol 2004; 13: 183-189.

12. Chen CJ, Jeng L Bin, Huang SF. Lymphoepithelioma-like hepatocellular carcinoma. Chang Gung Med $J$ 2007; 30: 172-177.

13. Nemolato S, Fanni D, Naccarato AG, Ravarino A, Bevilacqua G, Faa G. Lymphoepitelioma-like hepatocellular carcinoma: A case report and a review of the literature. World J Gastroenterol 2008; 14: 4694-4696.

14. Park HS, Jang KY, Kim YK, Cho BH, Moon WS. Hepatocellular carcinoma with massive lymphoid infiltration: A regressing phenomenon? Pathol Res Pract 2009; 205: 648-652.

15. Shinoda M, Kadota Y, Tsujikawa H, et al. Lymphoepithelioma-like hepatocellular carcinoma: A case report and a review of the literature. World J Surg Oncol 2013; 11: 97.

16. Patel KR, Liu TC, Vaccharajani N, Chapman WC, Brunt EM. Characterization of inflammatory (lymphoepithelioma-like) hepatocellular carcinoma: A study of 8 cases. Arch Pathol Lab Med 2014; 138: 1193-1202.

17. An SL, Liu LG, Zheng YL, et al. Primary lymphoepithelioma-like hepatocellular carcinoma: Report of a locally advanced case and review of literature. Int J Clin Exp Pathol 2015; 8: 3282-3287.

18. Chan AWH, Tong JHM, Pan Y, et al. Lymphoepithelioma-like hepatocellular carcinoma: An uncommon variant of hepatocellular carcinoma with favorable outcome. Am J Surg Pathol 2015; 39: 304-312. 
19. Insilla AC, Faviana P, Pollina LE, et al. Lymphoepithelioma-like hepatocellular carcinoma: Case report and review of the literature. World J Gastroenterol 2015; 21: 10468-10474.

20. Wei J, Liu Q, Wang C, Yu S. Lymphoepithelioma-like hepatocellular carcinoma without Epstein-Barr virus infection: A case report and a review of the literature. Indian J Pathol Microbiol 2015; 58: 550-553.

21. Miyasaka C, Ishida M, Ito H, Kaibori M, Uemura Y, Tsuta K. Lymphoepithelioma-like hepatocellular carcinoma: A case report with emphasis on the cytological features. Int J Clin Exp Pathol 2017; 10: 7893-7897.

22. Wang JK, Jin YW, Hu HJ, et al. Lymphoepithelioma-like hepatocellular carcinoma. A case report and brief review of literature. Medicine2017; 96: e9416.

23. Shih EJ, Chau IY, Yeh YC, Chau GY. Synchronous hepatocellular carcinoma and lymphoepitheliomalike carcinoma arising from 2 different sites of the liver: A case report. Medicine 2018; 97: e12548.

24. Filotico M, Moretti V, Floccari F, D'Amuri A. Very Rare Liver Neoplasm: Lymphoepithelioma-Like (LEL) Hepatocellular Carcinoma .Case Rep Pathol 2018; 2018: 2651716.

25. Finn RS, Qin S, Ikeda M, et al. Atezolizumab plus Bevacizumab in Unresectable Hepatocellular Carcinoma. N Engl J Med 2020; 382: 1894-1905.

26. Chan AWH, Zhang Z, Chong CCN, Tin EKY, Chow C, Wong N. Genomic landscape of lymphoepithelioma-like hepatocellular carcinoma. J Pathol 2019; 249: 166-172.

27. Kim C, Rajan A, DeBrito PA, Giaccone G. Metastatic lymphoepithelioma-like carcinoma of the lung treated with nivolumab: A case report and focused review of literature. Translational Lung Cancer Research 2016; 5: 720-726.

28. Kumar V, Dave V, Harris J, Huang Y. Response of advanced stage recurrent lymphoepithelioma-like carcinoma to nivolumab.Immunotherapy 2017; 9: 955-961.

29. Calderaro J, Rousseau B, Amaddeo G, et al. Programmed death ligand 1 expression in hepatocellular carcinoma: Relationship with clinical and pathological features. Hepatology 2016; 64: 2038-2046.

30. Hartley GP, Chow L, Ammons DT, Wheat WH, Dow SW. Programmed cell death ligand 1 (PD-L1) signaling regulates macrophage proliferation and activation. Cancer Immunology Research 2018; 6: 12601273 .

\section{Figure legends}

Figure 1. Abdominal ultrasonography images. The tumor was present on segment 5 and showed a mosaic pattern (white arrow).

Figure 2. CT during angiography. The tumor showed low absorption on CT during arterial portography (a) and high absorption on CT during hepatic arteriography both in the early phase (b) and late phase (c).

Figure 3. EOB-MRI images. The tumor showed a high signal on early phase (a), low signal on T1-weighted hepatocyte phase (b), high signal on diffusion-weighted imaging (c), and low signal on apparent diffusion coefficient (d).

Figure 4. Histological findings in resected lymphocyte rich hepatocellular carcinoma (loupe image, H-E stain). The enlarged image is shown in the black frame. White line shows the border of the tumor and liver background. H-E staining showed poorly differentiated tumor cells with prominent nuclear irregularities and high degree of lymphocytic infiltration in the background.

Figure 5. H-E staining and immunostaining images of tumor. H-E (a), Glypican3 (b), Hepatocyte (c), EBV-encoded small RNA (EBER) (d). Many infiltrating lymphocytes were found around tumor cells stained with Glypican3 and Hepatocyte. EBER was negative. 
Figure 6. Immunostaining images of infiltrating lymphocytes. CD8 (a), CD4 (b), CD3 (c), CD20 (d). Both CD8 and CD4 were positive in 50\%. CD3 was positive in almost $100 \%$ and CD20 was negative.

Figure 7. Immunostaining images. FOXP3 (a), Programmed cell death 1 (PD-1) (b), Programmed cell death ligand 1 (PD-L1) (c). FOXP3 was weakly positive in CD4 positive cells. PD-1 was positive in almost all lymphocytes. PD-L1 was positive in about $40 \%$ of tumor cells.

Figure 8. Low-power field showing immunostaining of PD-L1 (a). High-power field showing Glypican 3 immunostaining (b) and PD-L1 (c). Multiple small foci of PD-L1 positive inflammatory cell clusters were founded around Glypican 3-positive tumor cells (circles).

Tables

Table 1. Laboratory data on admission.

Table 2. The 59 cases of poorly differentiated HCC, lymphocyte-rich.

We searched PubMed for articles from 1995 to 2019 using the keywords "lymphoepithelioma-like hepatocellular carcinoma" or "hepatocellular carcinoma lymphocyte rich". As a result, 58 cases of poorly differentiated HCC, lymphocyte-rich were identified. The table shows a total of 59 cases, including the current case reported.

\section{Hosted file}

Figure.pptx available at https://authorea.com/users/392594/articles/524806-a-case-of-poorlydifferentiated-hepatocellular-carcinoma-lymphocyte-rich

\section{Hosted file}

Table.xlsx available at https://authorea.com/users/392594/articles/524806-a-case-of-poorlydifferentiated-hepatocellular-carcinoma-lymphocyte-rich 